\title{
Management of hydrocephalus and subdural hygromas in pediatric patients after decompression of Chiari malformation type I: case series and review of the literature
}

\author{
${ }^{*}$ Andrew C. Vivas, MD, ${ }^{2}$ Nir Shimony, MD, ${ }^{1}$ Eric M. Jackson, MD, ${ }^{3}$ Risheng Xu, MD, ${ }^{3}$ \\ George I. Jallo, MD, ${ }^{1,3}$ Luis Rodriguez, MD, ${ }^{1}$ Gerald F. Tuite, MD, ${ }^{1}$ and Carolyn M. Carey, MD, MBA ${ }^{1}$ \\ 1Department of Neurosurgery, Johns Hopkins All Children's Institute for Brain Protection Sciences, St. Petersburg; ${ }^{2}$ Department \\ of Neurosurgery, University of South Florida, Tampa, Florida; and ${ }^{3}$ Department of Neurosurgery, Johns Hopkins University \\ School of Medicine, Baltimore, Maryland
}

\begin{abstract}
OBJECTIVE Hydrocephalus associated with subdural hygromas is a rare complication after decompression of Chiari malformation type I (CM-I). There is no consensus for management of this complication. The authors present a series of 5 pediatric patients who underwent CM-I decompression with placement of a dural graft complicated by posterior fossa hygromas and hydrocephalus that were successfully managed nonoperatively.
\end{abstract}

METHODS A retrospective review over the last 5 years of patients who presented with hydrocephalus and subdural hygromas following foramen magnum decompression with placement of a dural graft for CM-I was conducted at 2 pediatric institutions. Their preoperative presentation, perioperative hospital course, and postoperative re-presentation are discussed with attention to their treatment regimen and ultimate outcome. In addition to reporting these cases, the authors discuss all similar cases found in their literature review.

RESULTS Over the last 5 years, the authors have encountered 194 pediatric cases of CM-I decompression with duraplasty equally distributed at the 2 institutions. Of those cases, 5 pediatric patients with a delayed postoperative complication involving hydrocephalus and subdural hygromas were identified. The 5 patients were managed nonoperatively with acetazolamide and high-dose dexamethasone; dosages of both drugs were adjusted to the age and weight of each patient. All patients were symptom free at follow-up and exhibited resolution of their pathology on imaging. Thirteen similar pediatric cases and 17 adult cases were identified in the literature review. Most reported cases were treated with CSF diversion or reoperation. There were a total of 4 cases previously reported with successful nonoperative management. Of these cases, only 1 case was reported in the pediatric population.

CONCLUSIONS De novo hydrocephalus, in association with subdural hygromas following CM-I decompression, is rare. This presentation suggests that these complications after posterior fossa decompression with duraplasty can be treated with nonoperative medical management, therefore obviating the need for CSF diversion or reoperation.

https://thejns.org/doi/abs/10.3171/2018.4.PEDS17622

KEYWORDS Chiari I malformation; complication; hydrocephalus; posterior fossa decompression; subdural hygromas

$\mathrm{D}$ ESCENT of the cerebellar tonsils greater than $5 \mathrm{~mm}$ below the level of the foramen magnum, known as Chiari malformation type I (CM-I), is a wellrecognized entity in both adult and pediatric patients. ${ }^{17,22}$ It is generally accepted that suboccipital craniectomy with a $\mathrm{C} 1$ laminectomy, with or without dural expansion, is the standard of care for patients with symptomatic primary CM-I. $1,8,10,14,30$ This is especially true with an associated syrinx, which may serve as an indicator of abnormal CSF dynamics. ${ }^{2,18}$ While techniques for the decompression may vary from surgeon to surgeon, the creation of space at the cervicomedullary junction, with improvement of CSF flow

ABBREVIATIONS CM-I = Chiari malformation type I; ETV = endoscopic third ventriculostomy; $F M D=$ foramen magnum decompression. SUBMITTED December 2, 2017. ACCEPTED April 17, 2018.

${ }^{*}$ A.C.V. and N.S. contributed equally to this work. 
obstruction and brainstem compression, is the accepted general goal of surgery. Common complications following CM-I decompression include CSF leakage, pseudomeningocele, meningitis (bacterial or chemical), postoperative hemorrhage, and cerebellar ptosis or slump..$^{2}$ Less common complications include vascular injury and stroke. ${ }^{20}$ Subdural hygromas and postoperative hydrocephalus after posterior fossa decompression are rare but recognized complications without a consensus for management. ${ }^{4-6,9,11,19,23,24,26,27,31}$ A variety of treatments have been described, including temporary CSF diversion, permanent CSF diversion, endoscopic third ventriculostomy (ETV), reoperation to widen the arachnoidal opening, and evacuation of subdural hygromas..$^{24,27,31}$ There are rare reports of good outcomes after nonoperative management. ${ }^{9,11,19}$ We present our experience with 5 pediatric patients at 2 institutions and discuss nonoperative strategies for treatment. Additionally, we present a review of pediatric and adult cases of CM-I complicated by hydrocephalus with subdural hygromas that have been reported in the literature and discuss the possible pathogenesis and treatment of this rare complication.

\section{Methods}

At both pediatric institutions, a retrospective review of the last 5 years was done in order to identify operative cases of CM-I decompression with duraplasty. Data were obtained identifying patients who presented with hydrocephalus and subdural hygromas. For the purposes of this study, we defined CM-I as tonsillar herniation greater than $5 \mathrm{~mm}$ below the foramen magnum with presenting symptoms attributable to the malformation. Five pediatric patients (age 1.5-17 years, average 8.9 years) were identified from the database. IRB approval was obtained for purposes of this review. The patients were those who underwent foramen magnum decompression (FMD) for $\mathrm{CM}-\mathrm{I}$, and whose postoperative courses were complicated by delayed postoperative subdural fluid collections (either cerebellar or cerebral) and evidence of intracranial hypertension and enlarged ventricles. Preoperative, operative, and postoperative records for these cases were reviewed. Asymptomatic patients and patients with CM-I attributable to other causes, such as mass lesion, hydrocephalus, spinal cord tethering, and other secondary type I Chiari malformations (e.g., from lumboperitoneal shunting or intracranial hypotension), were excluded. Table 1 contains the summary for our patients' data.

\section{Operative Technique}

Our cohort of pediatric patients underwent surgery performed by 4 pediatric neurosurgeons at 2 pediatric neurosurgical centers. The operations were performed with the patient in the prone position. A midline cervical dissection was used to expose the occiput and high cervical spine. Suboccipital craniectomy with $\mathrm{C} 1$ laminectomy was performed to decompress the foramen magnum. The dura and arachnoid maters were then opened. In 4 of our patients, the cerebellar tonsils were cauterized to shrink and elevate them. In all cases, a thorough arachnoidolysis was done, with specific attention to remove any veil or tissue webs that could potentially obstruct the CSF pathway around the outlet of the fourth ventricle. Careful arachnoidolysis was also done laterally toward the gutters around the tonsils in order to release any potential arachnoid pockets. An autograft dural graft (pericranium) was used in 3 cases, whereas allograft dural graft was used in the remaining 2 cases. The graft was sutured into place in order to enlarge the intradural diameter at the craniovertebral junction $(\mathrm{CVJ})$ and prevent further compression of the intradural contents at the CVJ. The patched dura and subsequently the overlying fascia were both closed in a watertight fashion. Operative goals and immediate postoperative results were equivalent despite minor technical differences due to surgeon preferences. Intraoperative ultrasound was not routinely used.

\section{Literature Review}

MEDLINE and PubMed searches were performed using the keywords "Chiari malformation," "foramen magnum decompression," "hydrocephalus," "subdural hematoma," "subdural hygroma," "cerebellar ptosis," "cerebellar sag," and "cerebellar slump." All articles discussing postoperative intracranial hypertension with subdural fluid collections (either cerebellar or cerebral) as a complication following posterior fossa decompression, along with respondent commentaries regarding these articles, were included. We extracted pediatric case data from the published case reports and case series for comparison and analysis. Adult data are presented for a thorough and complete analysis but are not considered in our analysis, as this report focuses on a pediatric population.

\section{Case Presentations}

Table 1 contains a summary of our patients' data.

\section{Case 1}

This 14-year-old girl with CM-I and syringomyelia presented with a 3-month history of pain and numbness moving up the left upper limb to the left back, chest, and neck in a hemicape-like distribution. Neurological examination revealed hypesthesia to temperature and pin sensation in the left upper extremity down to approximately the T4 level (slightly beyond midline) as well as to the posterior neck and head. All other examination findings were normal. MRI revealed a CM-I with cerebellar tonsils terminating $6 \mathrm{~mm}$ below the foramen magnum, as well as a large cervicothoracic syrinx extending from $\mathrm{C} 1$ to $\mathrm{T} 8$ and deviating eccentrically to the left side. Of note, no hydrocephalus or ventriculomegaly was evident at admission (Fig. 1A).

The patient underwent a standard posterior fossa decompression as previously described. The arachnoid was widely opened, and the obex was visualized without any signs of obstructing tissue or web. The tonsils were not cauterized. Blood loss was estimated to be $75 \mathrm{ml}$. An allograft dural graft was utilized for the duraplasty, which was closed in a watertight fashion. The patient was discharged home in good condition after an uneventful 3 -day postoperative course. Fourteen days after surgery, the patient developed headache, malaise, nausea, vomit- 


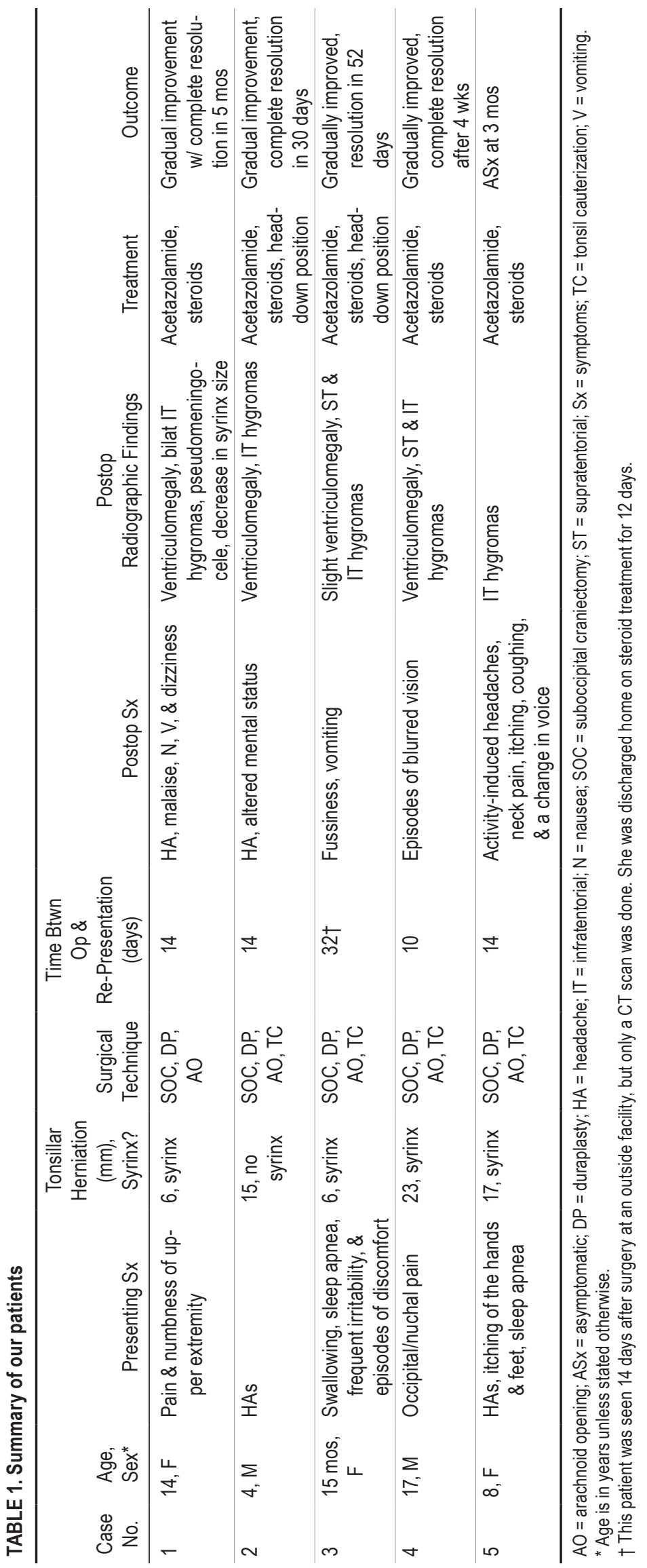



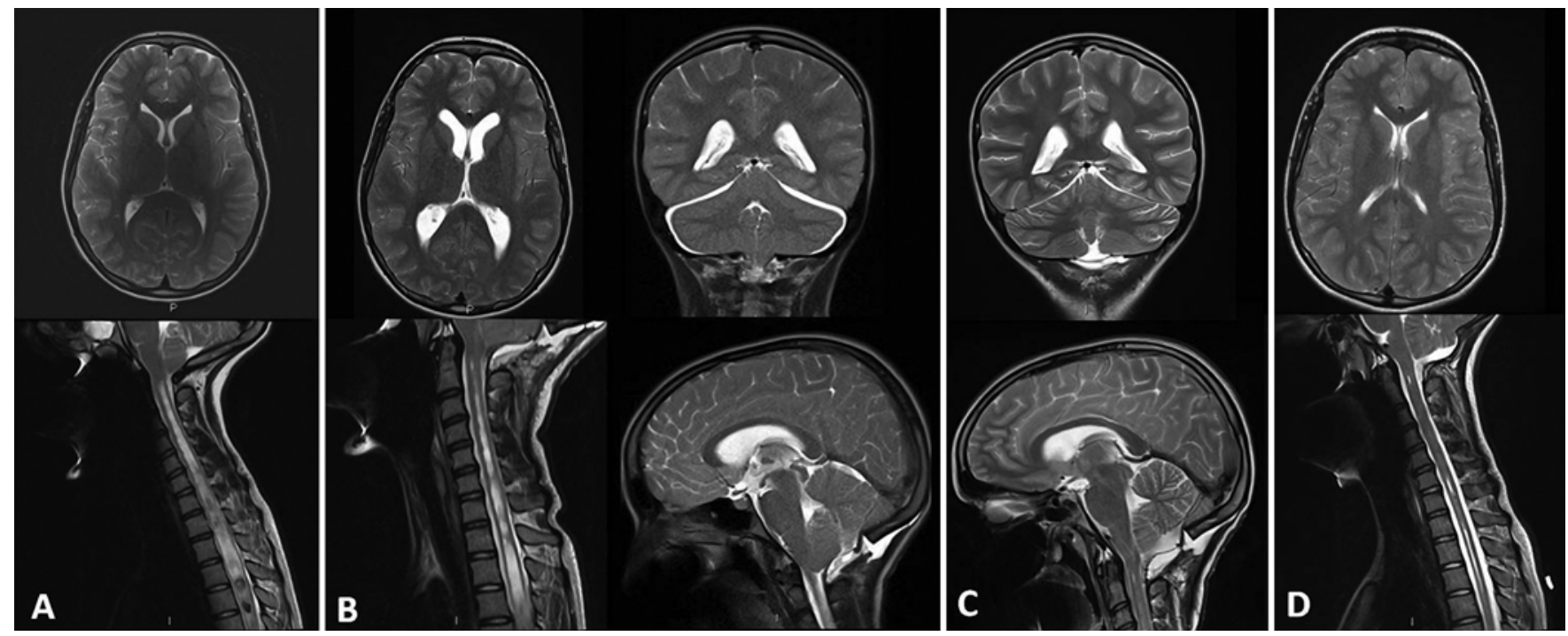

FIG. 1. Case 1. Axial, sagittal, and coronal T2-weighted MRI scans obtained pre-FMD (A), and 14 days (B), 21 days (C), and 5 months (D) post-FMD, showing the progressive appearance and decrease of bilateral infratentorial subdural fluid collections in addition to normalization of hydrocephalus.

ing, and dizziness on cervical rotation. She presented to a local emergency department a day after onset of symptoms where a lumbar puncture was performed to rule out meningitis. CSF results were without signs for suspected infectious process, and results of the final culture were negative. Her symptoms improved after the lumbar puncture. The patient was transferred to our facility for further evaluation. On examination, there was no evidence of CSF leak, wound discharge, or papilledema. Her neurological examination findings were normal aside from residual left upper extremity hypesthesia. MRI of the brain revealed ventriculomegaly consistent with hydrocephalus, bilateral infratentorial extraaxial fluid collections, and pseudomeningocele (Fig. 1B). There appeared to be a slight decrease in the size of the syrinx. A conservative treatment regimen (high-dose dexamethasone for 2 weeks, acetazolamide for 1 month) was initiated, with gradual alleviation of the patient's symptoms. She was monitored in the hospital for 7 days with repeat imaging that demonstrated mild improvement (Fig. 1C). Complete resolution of ventriculomegaly was noted 5 months later, with continued improvement of left posterior head numbness and residual syrinx (Fig. 1D).

\section{Case 2}

This 4-year-old boy was complaining at presentation of headaches several times a week for several months. His caregivers described him holding his neck and the back of his head complaining of pain. He also had episodes of irritability and temper outbursts but at other times would be quite agreeable and complacent. Staring events and stiffening of limbs during sleep were reported, yet 48-hour electroencephalography demonstrated no epileptic activity. He also had mild speech delay.

MRI was performed, which demonstrated a CM-I with $15 \mathrm{~mm}$ of tonsillar herniation below the foramen magnum and an impacted foramen magnum. There was no evidence of ventriculomegaly or syrinx on preoperative imaging (Fig. 2A).

The patient underwent posterior fossa decompression with a laminectomy of $\mathrm{Cl}$. The fourth ventricle and the region of the obex were inspected, and extensive arachnoidolysis was performed. There was no scarring or arachnoidal veil visible. The tonsils were very large, extending to $\mathrm{C} 2$, and they were cauterized. Blood loss was minimal and estimated around $15 \mathrm{ml}$. A pericranial graft was utilized for the duraplasty, which was closed in a watertight fashion. The muscle, fascia, and subcutaneous tissues were also tightly closed. The patient did well postoperatively and returned home 3 days after surgery. Fourteen days after surgery, he presented to a local hospital with a complaint of severe headache that woke him from sleep in the early morning hours. He had headaches for a few days before presentation, but they were not severe enough for his family to seek medical attention. The patient was then transferred to our facility. He was sleepy and complained of severe headache ( 9 of 10 on a numeric rating scale). His symptoms were worse while in a sitting position. He had no signs of infection. Of note, he had sustained a minor fall from his bike the day before admission. CT scans obtained at the local facility after the fall showed ventriculomegaly of the supratentorial ventricles and a normal-sized fourth ventricle as well as posterior fossa subdural hygromas. MRI performed at our institution better delineated these findings (Fig. 2B). The ophthalmology examination results were normal, without signs of papilledema.

The child was then placed in a head-down position and experienced immediate relief of his symptoms. He was started on acetazolamide and high-dose dexamethasone. He was able to sit up for periods of time on day 3 of treatment, with a significant decrease in headache symptoms. MRI done on day 2 after admission showed a mild decrease in ventricular size, as well as a decrease in the posterior fossa subdural fluid. By day 6 the patient was 

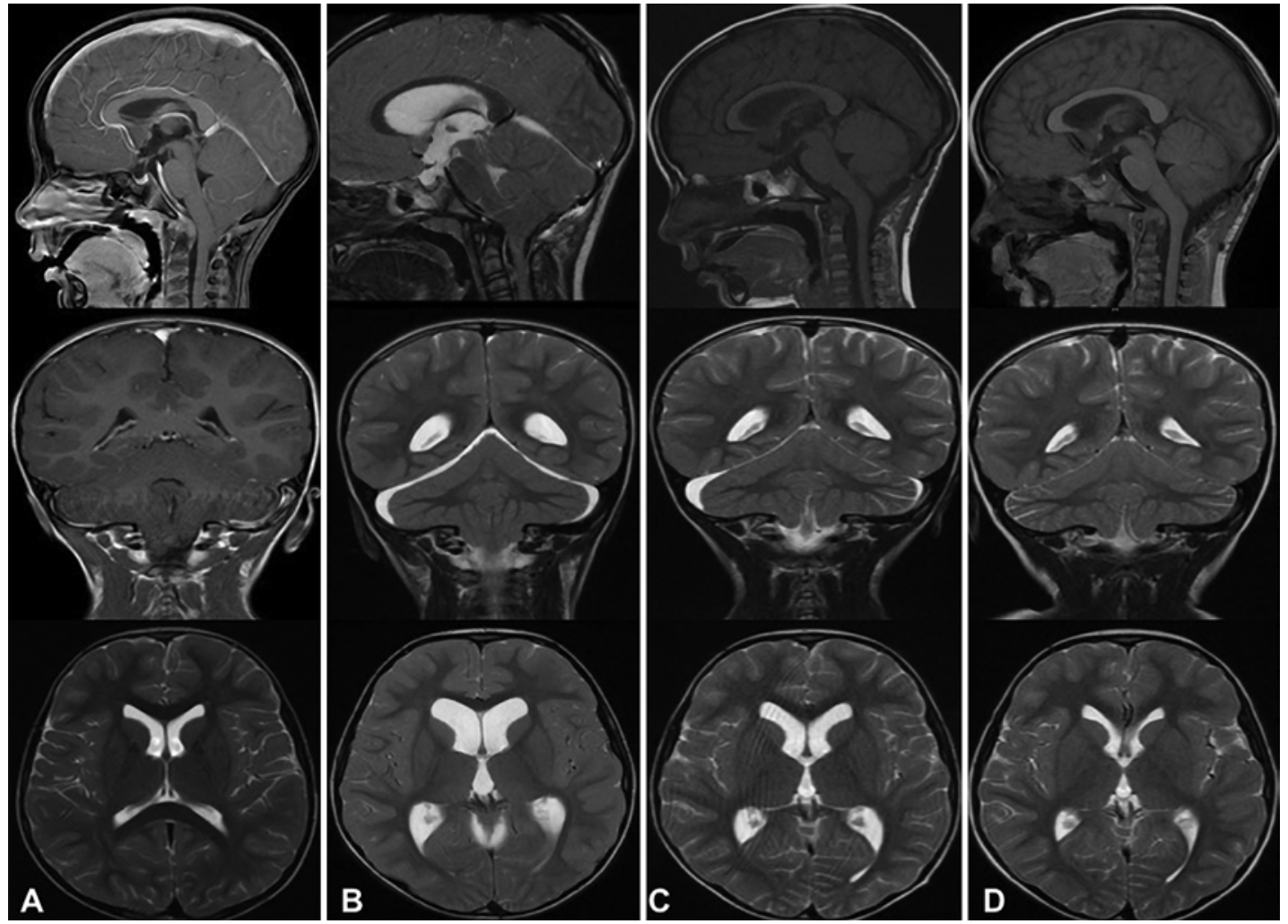

FIG. 2. Case 2. Sagittal, coronal, and axial T1- and T2-weighted MRI scans obtained pre-FMD (A), and 14 days (B), 21 days (C), and 1.5 months (D) post-FMD, showing the progressive appearance and decrease of bilateral infratentorial subdural fluid collections in addition to normalization of hydrocephalus.

ambulating and playing with no headaches. MRI done on day 7 showed a significant decrease in ventricular size and the subdural hygromas (Fig. 2C). A dexamethasone wean was started on day 6 and completed over a week. Acetazolamide continued for a total of 4 weeks with a gradual wean starting week 2 . The patient and his imaging continued to improve, and he returned to baseline 30 days after his presentation (Fig. 2D). His preoperative symptoms resolved, and his family felt he was more active.

\section{Case 3}

This 15-month-old girl presented with problems swallowing, sleep apnea, and frequent irritability and episodes of discomfort. MRI was done and showed a significant CM-I with about $6 \mathrm{~mm}$ of tonsillar descent below the foramen magnum. Preoperative MRI showed no signs of ventriculomegaly or syrinx (Fig. 3A). She underwent a posterior fossa decompression with laminectomy of $\mathrm{Cl}$, extensive arachnoidolysis, and cauterization of the cerebellar tonsils. Inspection of the obex confirmed no arachnoid veil or tissue obstructing the CSF flow from the fourth ventricle. A pericranial dural graft was utilized in order to close the dural opening in a watertight fashion. Blood loss was $10 \mathrm{ml}$. The patient did well postoperatively and was discharged home on postoperative day 2. Fourteen days after surgery, she was admitted to a regional facility. She presented with fussiness and had 1 episode of vomiting. Otherwise, she had no other complaints. The patient was started on dexamethasone for 12 days, and 4 days after weaning off the dexamethasone she was admitted again to our emergency department with complaints of irritability for several days, and again with 1 episode of emesis. There were no signs of infection or other viral illness. On examination, she had no focal neurological deficit and no sign of a pseudomeningocele.

The patient's MRI scan (32 days after surgery) showed extraaxial fluid collections around both cerebellar and cerebral convexities and along the interhemispheric fissure (Fig. 3B). There was a slight increase in the ventricle size. In comparison to her CT scan from 2 weeks prior, as reported by the other facility, MRI demonstrated an increase in the size of the extraaxial fluid supratentorially and infratentorially. She was started on acetazolamide, intravenous fluids, and high-dose dexamethasone and was positioned in bed with her head down. Three days after admission she felt much better and started to ambulate and play. On day 6 after presentation the repeat MRI showed almost complete resolution of the extraaxial fluid around the cerebrum and cerebellum (Fig. 3C). She clinically felt much better and was discharged home. When seen in follow-up her scanning results had returned to normal (Fig. 3D).

\section{Case 4}

This 17-year-old boy with developmental delay and known scoliosis was diagnosed with holocord syringomy- 

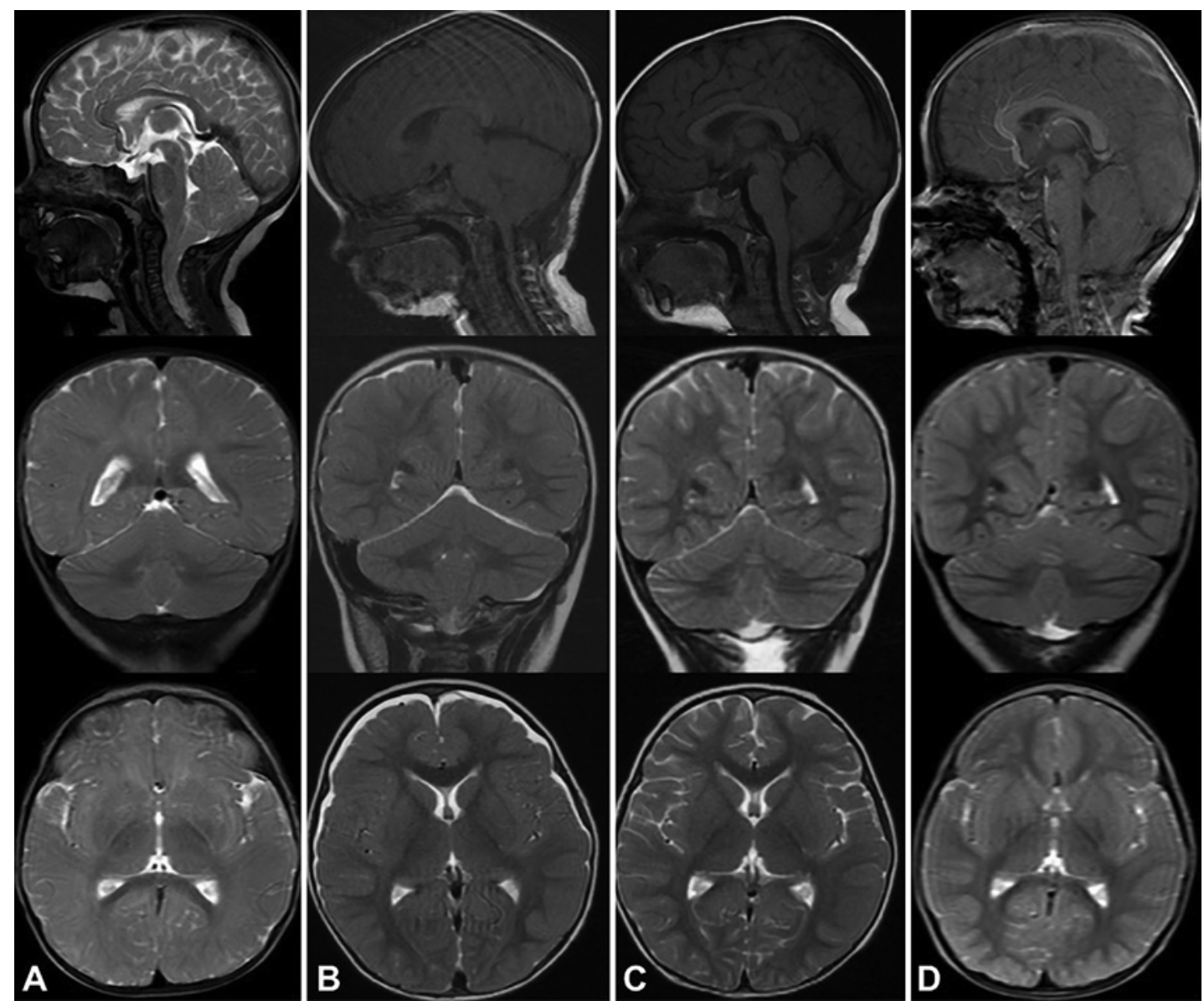

FIG. 3. Case 3. Sagittal, coronal, and axial T1- and T2-weighted MRI scans obtained pre-FMD (A), and 32 days (B), 38 days (C), and 52 days (D) post-FMD, showing the progressive appearance and decrease of bilateral infratentorial and supratentorial subdural fluid collections with gradual normalization.

elia and $23 \mathrm{~mm}$ of tonsillar ectopia while being assessed for his scoliosis with spinal imaging (Fig. 4A). On preoperative MRI there was no evidence of ventriculomegaly. A decision was made to take the patient to surgery for a posterior fossa decompression, laminectomy of $\mathrm{C} 1$, and duraplasty. Due to the low position of the tonsils, their fairly large size, and the anatomy at the foramen magnum, both cerebellar tonsils were cauterized. Thorough arachnoidolysis was done between the tonsils and lateral to the gutters around them. An inspection was done, revealing there was neither an arachnoid veil nor tissue obstructing the outlet of the fourth ventricle. Blood loss was $75 \mathrm{ml}$. A pericranial dural graft was utilized and installed in a watertight fashion.

Postoperatively the patient was treated with dexamethasone for 5 days. He experienced a few episodes of low oxygen saturation, which required administration of oxygen. No reason was found for the desaturation episodes. The patient did well and was discharged home 3 days after surgery. Ten days after surgery he presented to the emergency department with 2 episodes of binocular vision loss as reported by ophthalmology, without loss of consciousness, lethargy, headaches, or vomiting. An MRI of the brain demonstrated cerebellar hygromas and a slight increase in ventricle size with the formation of supratentorial and infratentorial hygromas as well (Fig. 4B). There was no apparent change in the position of the cerebellar tonsils. The patient had no evidence of papilledema on ophthalmological examination. He was started on acetazolamide and high-dose dexamethasone. Two days after admission and medical treatment, his episodes of blurred vision stopped, and his MRI findings improved with a minimal decrease in ventriculomegaly and a partial resolution of the subdural hygromas (Fig. 4C). The patient was discharged home on the medications and plans for further ambulatory follow-up. He was seen 2 weeks after discharge and had no new clinical symptoms. His imaging showed continued resolution of the hydrocephalus as well as complete resolution of the hygromas (Fig. 4D). He resumed his regular activities and was weaned from both dexamethasone and acetazolamide over the next 2 weeks.

\section{Case 5}

This 8-year-old girl had a known history of CM-I and syringomyelia of C4-T4. The patient had tonsillar decent of $17 \mathrm{~mm}$ below the foramen magnum. She had undergone 2 surgeries for foramen magnum decompression 5 years and 2 years prior to the current surgery. Her initial 

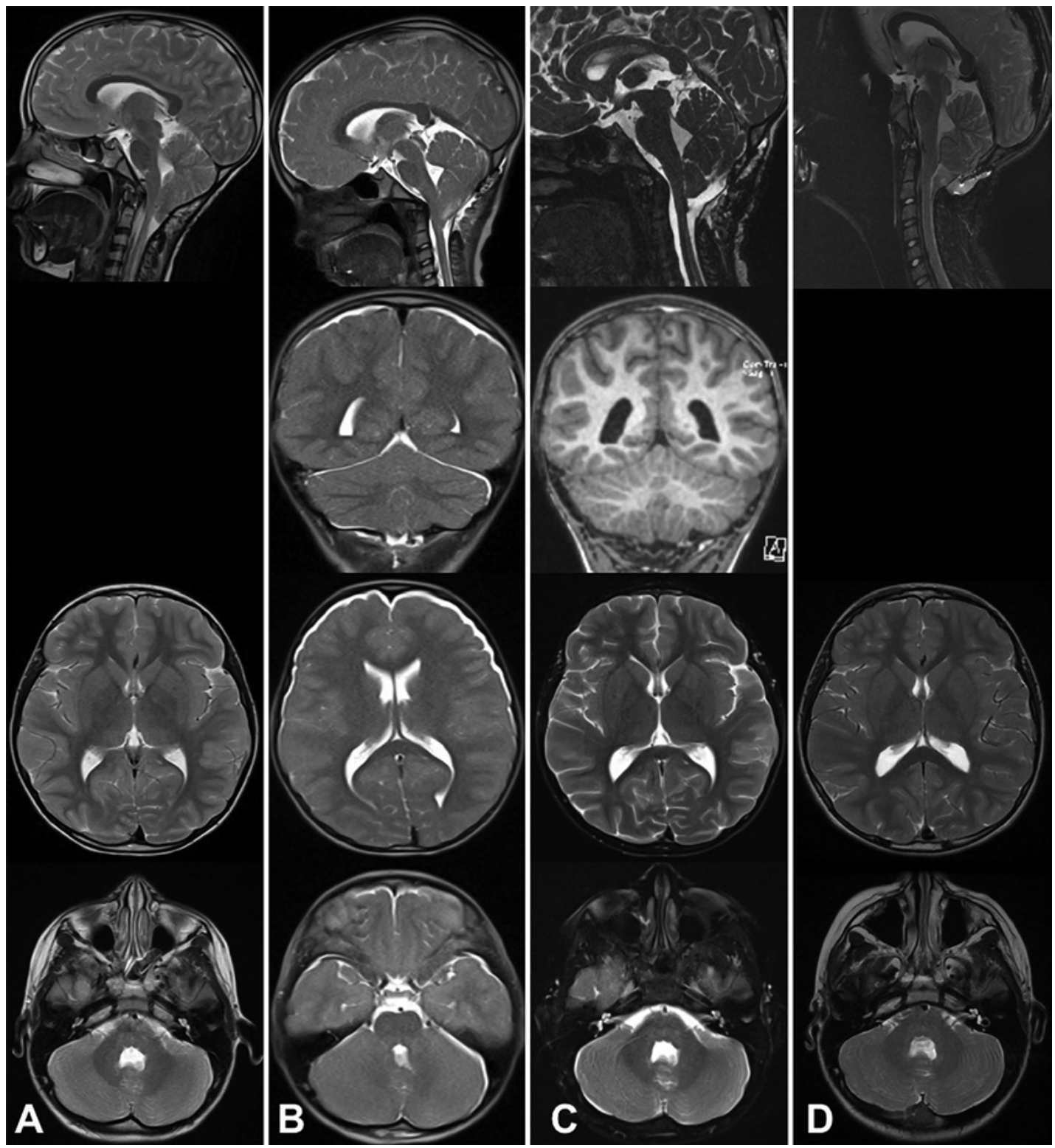

FIG. 4. Case 4. Sagittal, coronal, and axial T1- and T2-weighted MRI scans obtained pre-FMD (A), and 10 days (B), 12 days (C), and 28 days (D) post-FMD, showing the progressive appearance and decrease of bilateral infratentorial subdural fluid collections in addition to normalization of hydrocephalus.

presentation 5 years earlier was activity-induced occipital headaches and progressive itching of her hands and feet. MRI demonstrated the CM-I with an associated syrinx, and without evidence of ventriculomegaly. Foramen magnum decompression with duraplasty was performed. After the first surgery, she had resolution of her symptoms and remained asymptomatic for 3 years. She again developed activity-induced headaches, neck pain exacerbated by movement, itching in her legs, intermittent coughing, and a change in her voice. The patient underwent a second foramen magnum decompression surgery but with a smaller duraplasty this time. She was well for 2 years after that surgery when she again presented with similar symptoms, but this time with an additional diagnosis of central sleep apnea. She underwent a posterior fossa decompression, opening of scar tissue, extensive arachnoidolysis, cauterization of the tonsils, and a duraplasty using an allograft dural graft. Inspection of the obex revealed that there was no arachnoid veil or tissue obstructing the CSF pathways. Blood loss was estimated at $25 \mathrm{ml}$. After surgery, the patient initially did well but then developed a pseudomeningocele after severe coughing, which occurred 3 months after the decompression surgery. She presented with slightly enlarged ventricles (Fig. 5A) and eventually required exploratory surgery to repair the pseudomeningocele 5 months after the operation. Fourteen days after this surgery, the patient presented with headaches and an MRI demonstrated infratentorial hygromas and associated 
Vivas et al.

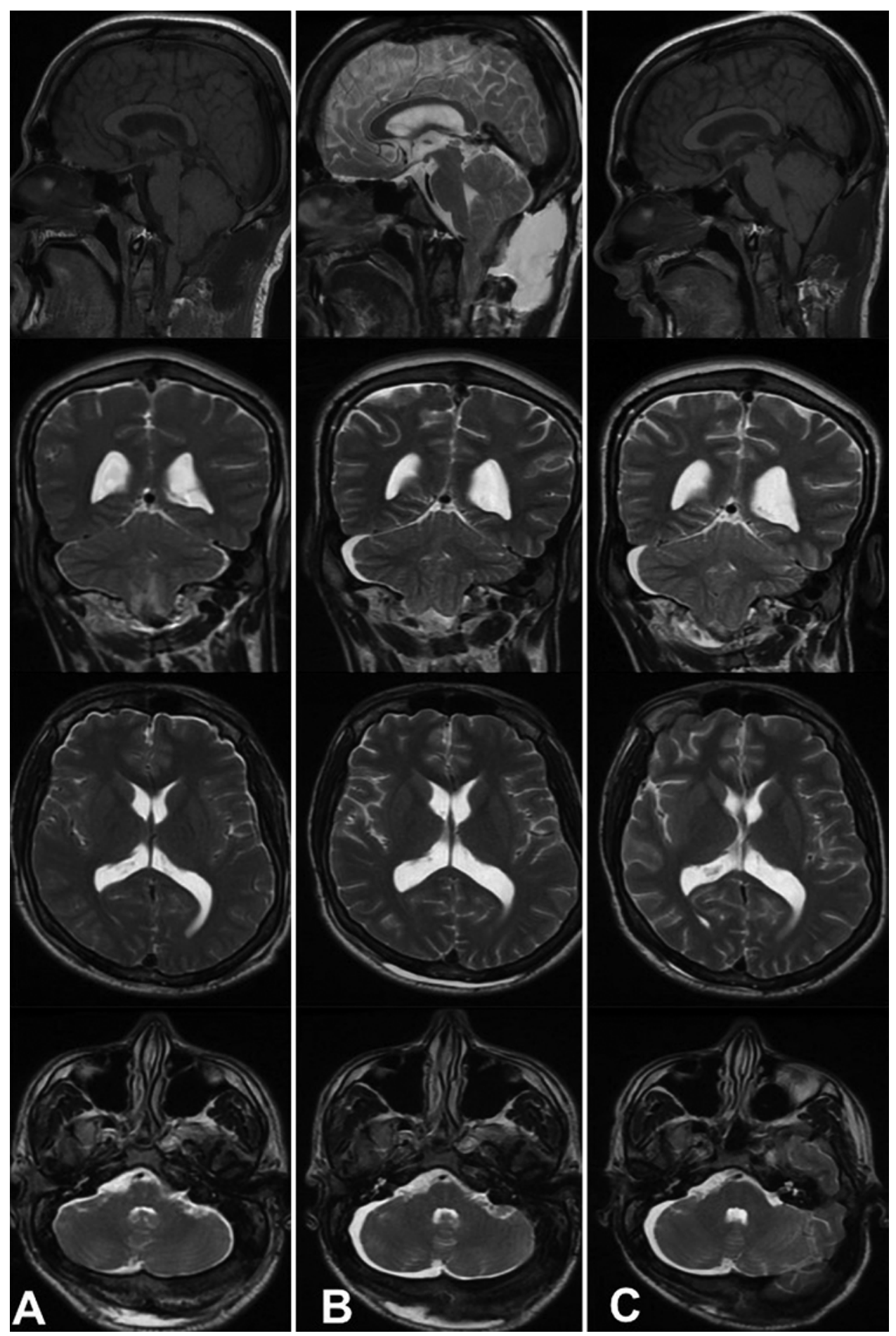

FIG. 5. Case 5. Sagittal, coronal, and axial T1- and T2-weighted MRI scans obtained post-FMD in the past and prefixation of pseudomeningocele (A), and 14 days after surgery with the formation of infratentorial hygromas (B), and 3 months after surgery with partial resolution in ventricular size and partial decrease of bilateral infratentorial subdural fluid collections (C).

mass effect (Fig. 5B). She was treated for several weeks with high-dose dexamethasone and a 2-week course of acetazolamide. She had slight resolution of the hygromas and significant improvement in her symptoms after 3 months (Fig. 5C).

\section{Literature Review}

The results of the literature search are shown in Tables 2 and 3. Table 2 includes all 13 published pediatric cases from 2001 to 2014. Zakaria et al. published the largest series of patients, which included 4 pediatric patients and 


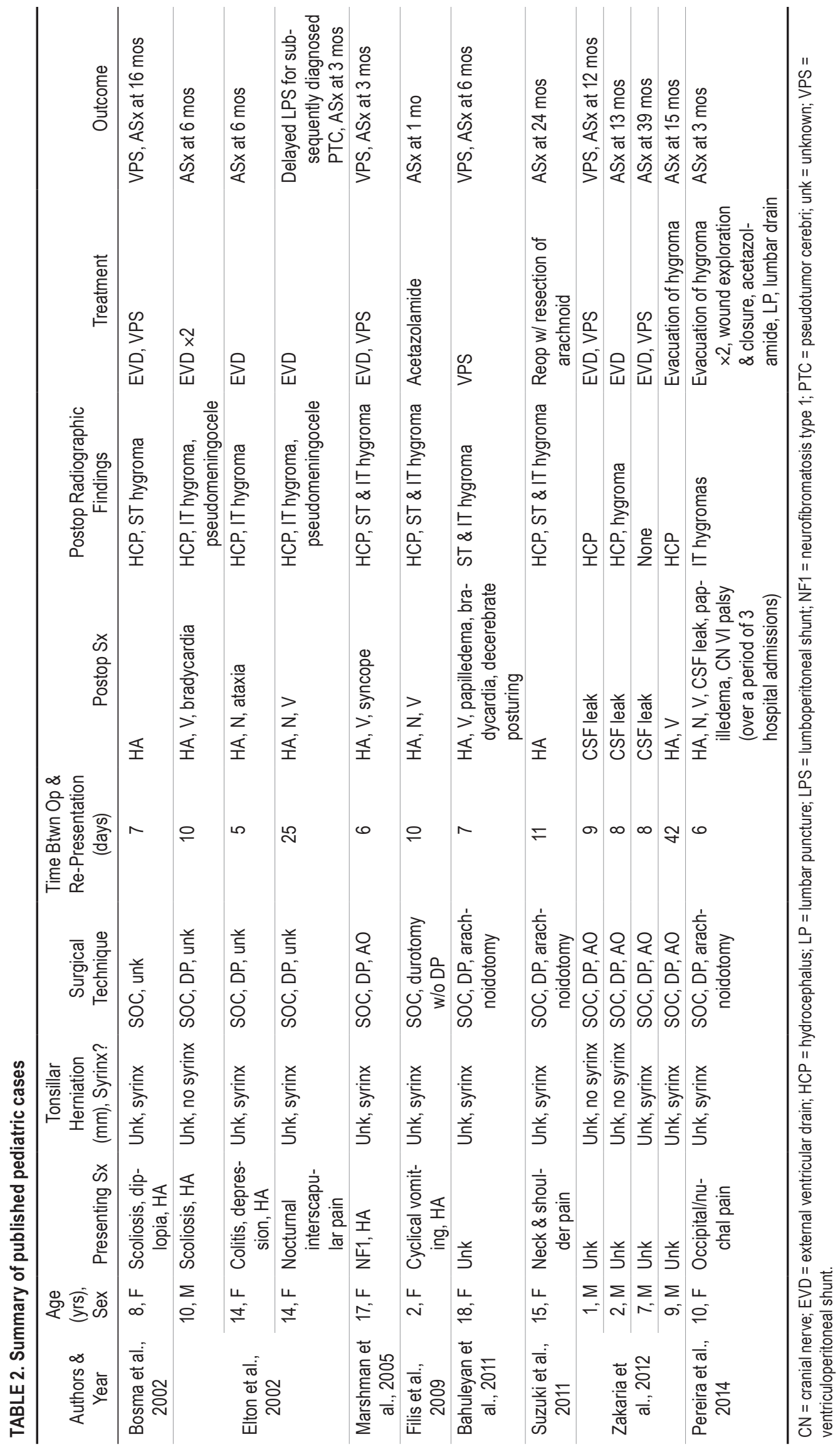



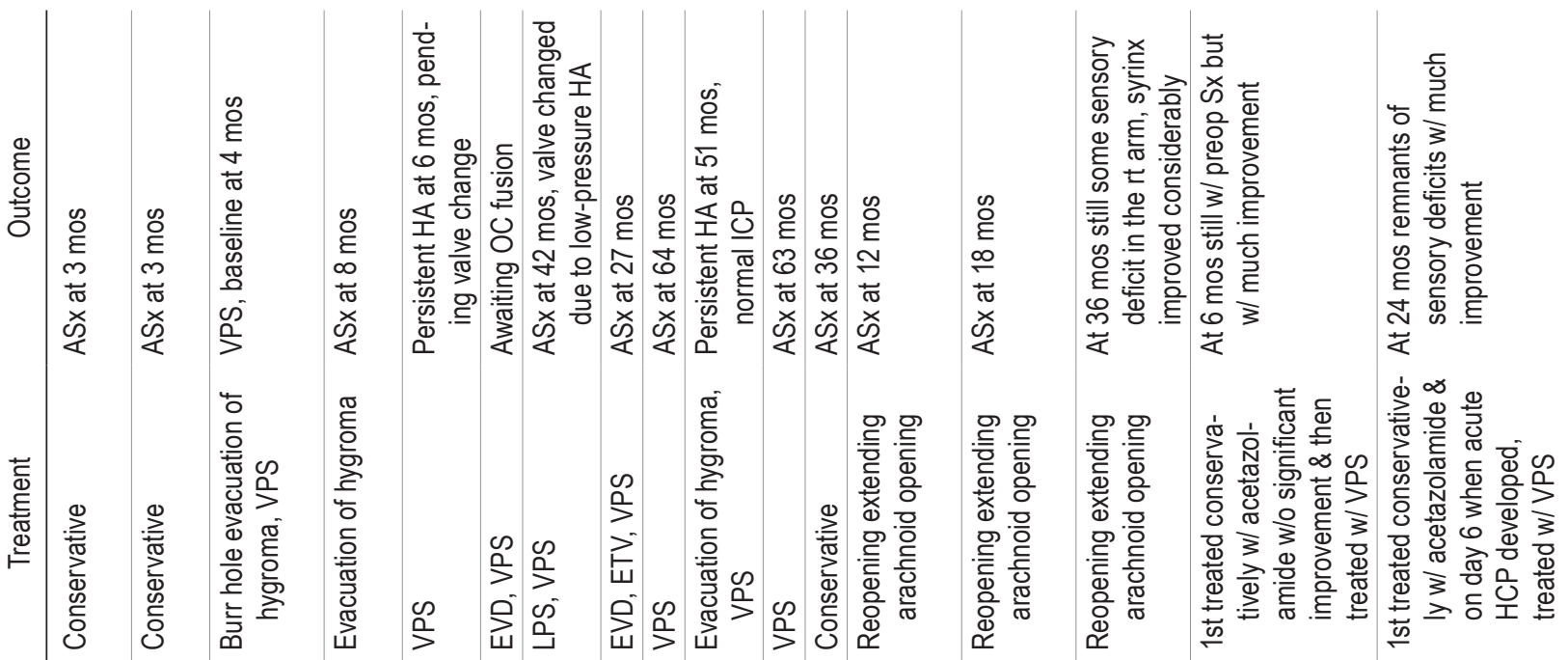

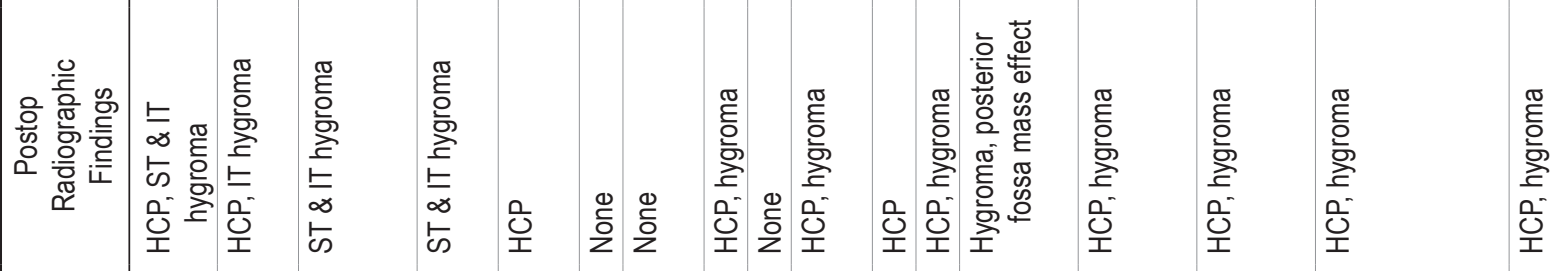

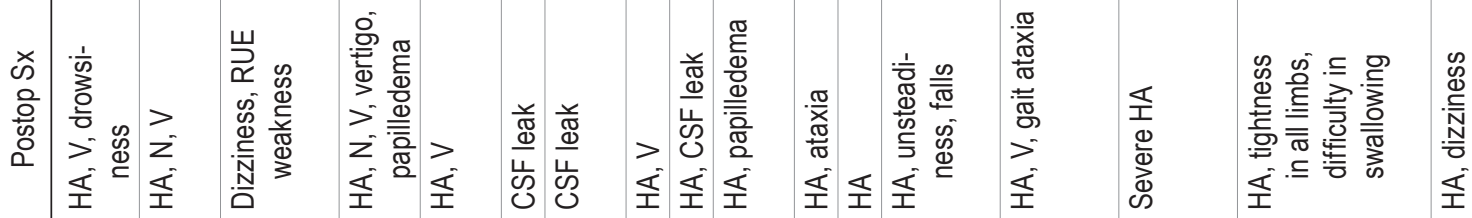
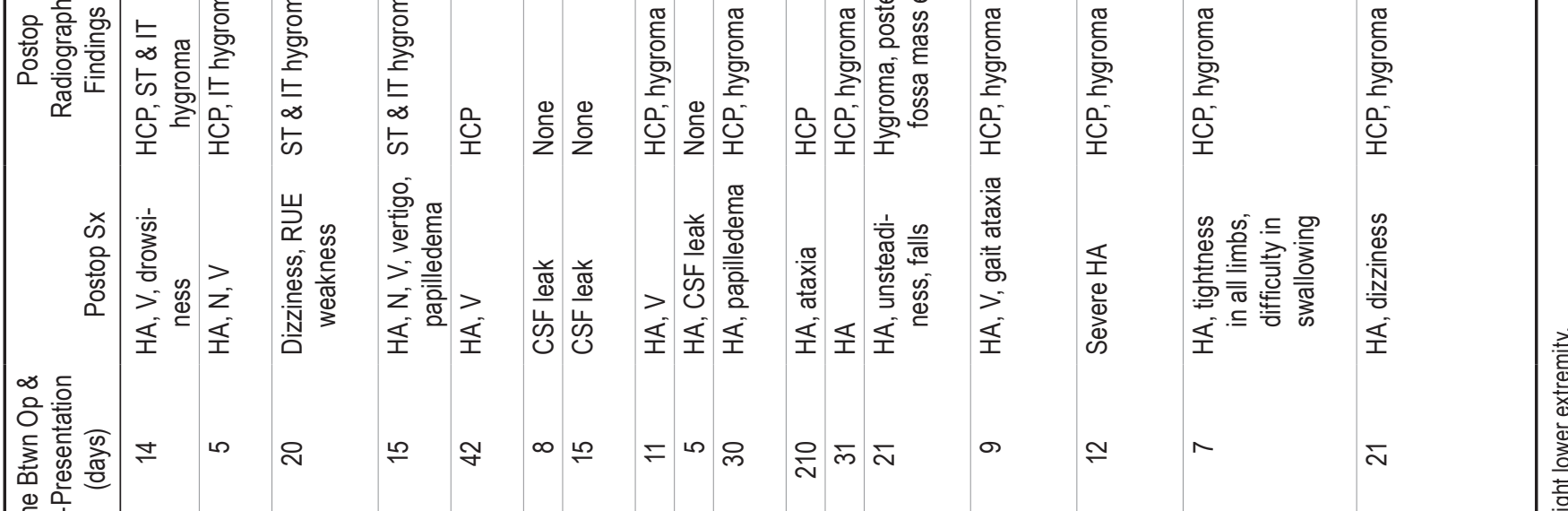

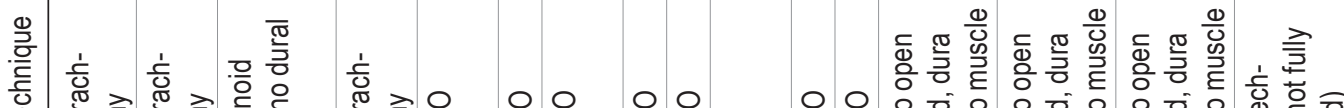

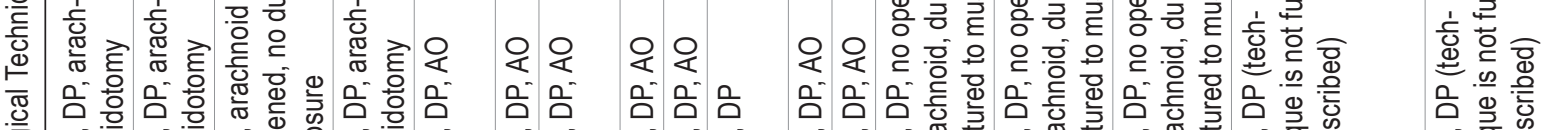

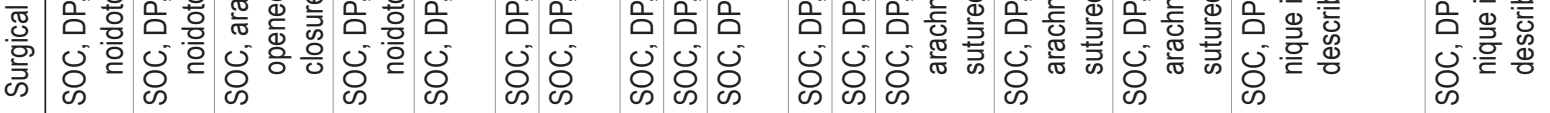

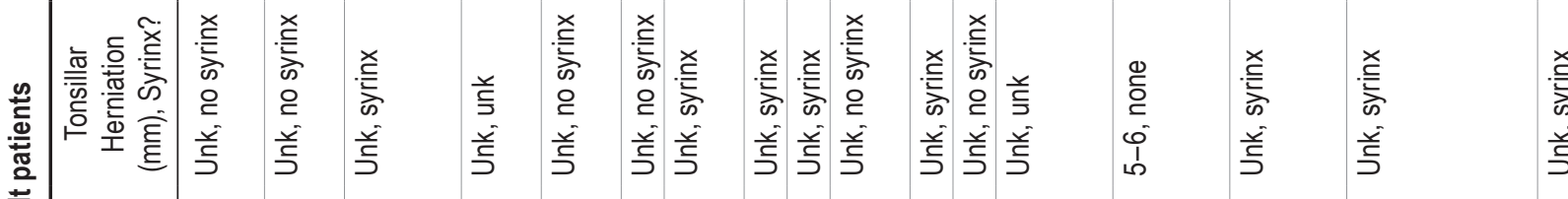

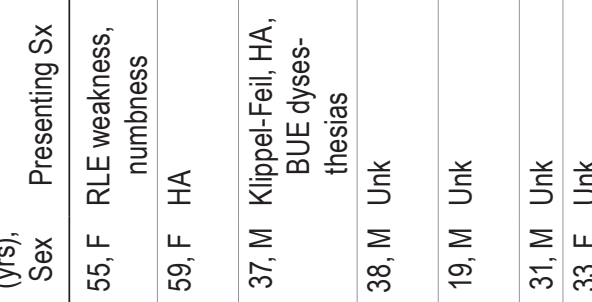

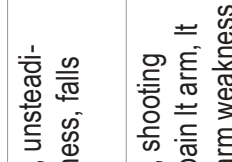

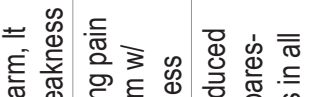

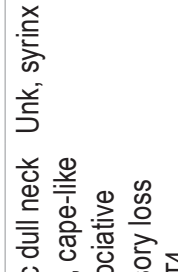

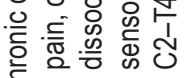
ป 인 
8 adult patients. ${ }^{31}$ The largest group of pediatric patients examined at one institution was by Elton et al., who reviewed 200 consecutive pediatric patients undergoing posterior fossa decompression and as such were able to publish information on the incidence of this complication. ${ }^{9}$ The age ranged from 1 to 18 years with a mean of 9.7 years. We were unable to determine the average length of tonsillar herniation based on published data, since different publications did not include the specific radiological description of every patient. Almost all pediatric patients in the published literature underwent suboccipital craniectomy and $\mathrm{C} 1$ laminectomy in combination with duraplasty and arachnoidal opening. On average, most of the patients presented again 11.8 days after surgery with a median of 8 days (range 5-42 days). In our series, patient age ranged from 15 months to 17 years with a mean of 8.8 years. The mean and median times to presentation were 13.25 and 14 days after surgery, respectively (range 10-32 days). Most patients reported on in the literature presented with headache, nausea, and vomiting, although 5 patients presented with more concerning symptoms of bradycardia or cranial neuropathy. ${ }^{5}$ One of these patients exhibited decerebrate posturing. While nonoperative management has been described by Filis et al., ${ }^{11}$ the other 12 patients were managed by CSF diversion (either temporarily via EVD or permanently via shunting) or operative reexploration. In the literature, the average length of follow-up was 11.3 months, with all patients being asymptomatic at last follow-up.

\section{Discussion}

Symptomatic primary CM-I is typically treated by posterior fossa decompression with the addition of a $\mathrm{C} 1$ laminectomy., ${ }^{4,510,17}$ There is much debate regarding the surgery for CM-I decompression, such as whether to perform bony decompression alone or to include additional steps, such as duraplasty, opening the arachnoid, or tonsillar cautery. $2,8,10,18,27$ Delayed postoperative subdural hygromas and acute hydrocephalus after CM-I decompression are rare complications but have been previously discussed in the literature. Most of the known literature focuses on the adult population. In the largest published series of pediatric CM-I decompressions, by Tubbs et al., 4 of 500 patients $(0.8 \%)$ developed this complication. ${ }^{29}$ Despite its rarity, most high-volume pediatric neurosurgical centers will likely encounter this complication, given the prevalence of symptomatic CM-I in the pediatric population.

Various mechanisms have been proposed to explain the pathophysiology of this complication. Some authors have theorized that small openings in the arachnoid at the time of surgery (either purposefully or incidentally) result in the formation of a 1-way slit-valve mechanism that allows CSF egress from the subarachnoid space into the subdural space. ${ }^{9,26}$ Some have suggested that the accumulated subdural fluid around the foramen magnum can potentially migrate with brain pulsation to the cisterna magna, which then compresses the cerebellar hemisphere either down toward the foramen magnum or anteriorly, compressing the aqueduct and fourth ventricle. ${ }^{5}$ Closure of the aqueduct (so-called "torqueing of the aqueduct") or closure of the foramina of Luschka and Magendie potentially can lead to obstructive hydrocephalus either above or below the fourth ventricle, with resulting clinical sequelae. Another potential explanation is related to cerebellar slump or sag, which is caused by the downward migration of the hindbrain after decompression of the foramen magnum. This descent of the cerebellum downward toward the foramen magnum in an already small posterior fossa in the pediatric population can potentially cause the partial obstruction of CSF pathways around the foramen magnum. ${ }^{3,7,15,16,25}$ In reviewing our cases, neither our radiologists nor we recognized any findings such as inflammation, the presence of hemorrhage, fourth ventricle obstruction, or diffuse edema that could potentially explain the findings of hydrocephalus or subdural hygromas.

In 2016, Guan and colleagues published an assessment of clinical risk factors for Chiari-related hydrocephalus in a cohort of 297 consecutive patients. ${ }^{13}$ They concluded that age younger than 6 years, a large amount of intraoperative blood loss, and the presence of fourth ventricular outlet obstruction are all associated with a greater chance for requiring long-term CSF diversion. In our cases, blood loss was minimal for those younger than 12 years and $75 \mathrm{ml}$ or less for older children. As mentioned in the description of the cases, in each case a thorough inspection for an arachnoid veil or web was done, and extensive arachnoidolysis was performed in order to prevent potential arachnoidal pockets or webs from obstructing the CSF pathways.

To date, there is no consensus regarding the best method to treat postoperative hydrocephalus associated with CM-I decompression. Several publications, describing either adult or pediatric patients, stress that an effort should be made to prevent this complication by both avoiding durotomy and arachnoidolysis whenever possible or, conversely, opening the arachnoid widely. Some authors have even suggested reoperation for closing the tiny hole in the arachnoid or opening the arachnoid widely and suturing it to the dura. ${ }^{9,26,31}$ However, in all of our cases, a wide arachnoidal opening was achieved but hydrocephalus was still observed, which leads to the possible conclusion that there are other alterations in CSF dynamics that may result in this pathology.

Currently, possible treatment options for post-CM-I decompression-associated hydrocephalus include conservative treatment, ventriculostomy, shunting, lumbar puncture, ETV, wound exploration for further decompression, and evacuation of hygromas. The overall prognosis, regardless of treatment modality, is excellent, with all reported patients achieving resolution of symptoms and radiographic abnormalities by 6 months (Tables $1-3$ ). The vast majority of pediatric patients reported with this complication were treated via temporary or permanent CSF diversion (9 of 13, or $69 \%$ ). ${ }^{9}$ Of the 4 patients managed without CSF diversion, 2 were managed by reoperation to widen the arachnoidal opening, and 1 was managed by evacuation of the subdural hygromas. Only 1 patient was managed nonoperatively. Among adult patients, most were treated with the use of CSF diversion (10 of 17, 58.9\%). Five patients were treated initially nonoperatively and 2 of them eventually did not improve and required CSF diversion (Table 3).

In review of our data, we identified an incidence of 
$2.6 \%$ (5/194 patients) for this condition. We add 5 pediatric cases to the literature that were managed nonoperatively and suggest that in patients without a precipitous decline in neurological function, a trial of observation and medical therapy is reasonable. All 6 pediatric patients managed nonoperatively ( 5 from our series and 1 reported by Filis et al. $)^{11}$ had excellent long-term outcomes and did not ultimately require CSF diversion. While this sample set is small, it appears clear that the postoperative hydrocephalus experienced by these patients is distinct from intrinsic increased intracranial hypertension that must be ruled out in the setting of CM-I prior to decompression (i.e., secondary Chiari malformation). We believe that the hydrocephalus is likely a result of altered CSF pathways by mass effect or inflammation around the surgical bed causing transient swelling of the tissue and obstructing CSF flow, eventually leading to the accumulation of cerebellar subdural hygromas. If the patient's neurological condition is stable, a trial of conservative, nonoperative treatment with acetazolamide and steroids, prescribed at the appropriate dose for weight, may eliminate the need for a more invasive therapy while natural CSF pathways are restored. Since most reported patients presented with classic symptoms of hydrocephalus, including headache, nausea, and vomiting, and only a minority of them presented with a deteriorating level of consciousness and symptoms of impending herniation (lethargy and bradycardia), we believe that the more aggressive treatment of CSF diversion solutions can be withheld in most of the cases while treating the patient conservatively.

\section{Conclusions}

De novo hydrocephalus associated with subdural hygromas is a known, albeit rare, complication after CM-I decompression with duraplasty. In carefully selected patients who are without signs or symptoms of impending herniation, it is reasonable to attempt nonoperative management.

\section{Acknowledgments}

We acknowledge the Johns Hopkins All Children's Research Foundation for providing financial support for this study.

\section{References}

1. Aitken LA, Lindan CE, Sidney S, Gupta N, Barkovich AJ, Sorel M, et al: Chiari type I malformation in a pediatric population. Pediatr Neurol 40:449-454, 2009

2. Alzate JC, Kothbauer KF, Jallo GI, Epstein FJ: Treatment of Chiari I malformation in patients with and without syringomyelia: a consecutive series of 66 cases. Neurosurg Focus 11(1):E3, 2001

3. Assina R, Meleis AM, Cohen MA, Iqbal MO, Liu JK: Titanium mesh-assisted dural tenting for an expansile suboccipital cranioplasty in the treatment of Chiari 1 malformation. J Clin Neurosci 21:1641-1646, 2014

4. Bahl A, Murphy M, Thomas N, Gullan R: Management of infratentorial subdural hygroma complicating foramen magnum decompression: a report of three cases. Acta Neurochir (Wien) 153:1123-1128, 2011

5. Bahuleyan B, Menon G, Hariharan E, Sharma M, Nair S: Symptomatic posterior fossa and supratentorial subdural hygromas as a rare complication following foramen magnum decompression for Chiari malformation Type I. J Neurosurg 114:510-513, 2011

6. Bosma JJD, Kumaran N, May PL: Cerebral oedema following EVD insertion for delayed hydrocephalus after foramen magnum decompression in Chiari I malformation. Childs Nerv Syst 18:474-477, 2002

7. Duddy MJ, Williams B: Hindbrain migration after decompression for hindbrain hernia: a quantitative assessment using MRI. Br J Neurosurg 5:141-152, 1991

8. Dyste GN, Menezes AH, VanGilder JC: Symptomatic Chiari malformations. An analysis of presentation, management, and long-term outcome. J Neurosurg 71:159-168, 1989

9. Elton S, Tubbs RS, Wellons JC III, Blount JP, Grabb PA, Oakes WJ: Acute hydrocephalus following a Chiari I decompression. Pediatr Neurosurg 36:101-104, 2002

10. Eule JM, Erickson MA, O’Brien MF, Handler M: Chiari I malformation associated with syringomyelia and scoliosis: a twenty-year review of surgical and nonsurgical treatment in a pediatric population. Spine (Phila Pa 1976) 27:1451-1455, 2002

11. Filis AK, Moon K, Cohen AR: Symptomatic subdural hygroma and hydrocephalus following Chiari I decompression. Pediatr Neurosurg 45:425-428, 2009

12. Furtado SV, Visvanathan K, Reddy K, Hegde AS: Pseudotumor cerebri: as a cause for early deterioration after Chiari I malformation surgery. Childs Nerv Syst 25:1007-1012, 2009

13. Guan J, Riva-Cambrin J, Brockmeyer DL: Chiari-related hydrocephalus: assessment of clinical risk factors in a cohort of 297 consecutive patients. Neurosurg Focus 41(5):E2, 2016

14. Hankinson T, Tubbs RS, Wellons JC: Duraplasty or not? An evidence-based review of the pediatric Chiari I malformation. Childs Nerv Syst 27:35-40, 2011

15. Heller JB, Lazareff J, Gabbay JS, Lam S, Kawamoto HK, Bradley JP: Posterior cranial fossa box expansion leads to resolution of symptomatic cerebellar ptosis following Chiari I malformation repair. J Craniofac Surg 18:274-280, 2007

16. Holly LT, Batzdorf U: Management of cerebellar ptosis following craniovertebral decompression for Chiari I malformation. J Neurosurg 94:21-26, 2001

17. Krieger MD, McComb JG, Levy ML: Toward a simpler surgical management of Chiari I malformation in a pediatric population. Pediatr Neurosurg 30:113-121, 1999

18. Madsen JR, Scott RM: Chiari malformations, syringomyelia, and intramedullary spinal cord tumors. Curr Opin Neurol Neurosurg 6:559-563, 1993

19. Marshman LA, Benjamin JC, Chawda SJ, David KM: Acute obstructive hydrocephalus associated with infratentorial subdural hygromas complicating Chiari malformation Type I decompression. Report of two cases and literature review. J Neurosurg 103: 752-755, 2005

20. Menezes AH: Chiari I malformations and hydromyeliacomplications. Pediatr Neurosurg 17:146-154, 1991-1992

21. Munakomi S, Bhattarai B, Chaudhary P: Case report: Acute obstructive hydrocephalus associated with infratentorial extra-axial fluid collection following foramen magnum decompression and durotomy for Chiari malformation type I. F1000 Res 5:33, 2016

22. Park JK, Gleason PL, Madsen JR, Goumnerova LC, Scott RM: Presentation and management of Chiari I malformation in children. Pediatr Neurosurg 26:190-196, 1997

23. Pereira EAC, Steele LF, Magdum SA: Recurrent subdural hygromas after foramen magnum decompression for Chiari Type I malformation. Br J Neurosurg 28:396-399, 2014

24. Perrini P, Rawlinson A, Cowie RA, King AT: Acute external hydrocephalus complicating craniocervical decompression for syringomyelia-Chiari I complex: case report and review of the literature. Neurosurg Rev 31:331-335, 2008 
25. Quigley MF, Iskandar B, Quigley ME, Nicosia M, Haughton V: Cerebrospinal fluid flow in foramen magnum: temporal and spatial patterns at MR imaging in volunteers and in patients with Chiari I malformation. Radiology 232:229-236, 2004

26. Ranjan A, Cast IP: Symptomatic subdural hygroma as a complication of foramen magnum decompression for hindbrain herniation (Arnold-Chiari deformity). Br J Neurosurg 10:301-303, 1996

27. Suzuki F, Kitagawa T, Takagi K, Nozaki K: Subacute subdural hygroma and presyrinx formation after foramen magnum decompression with duraplasty for Chiari type 1 malformation. Neurol Med Chir (Tokyo) 51:389-393, 2011

28. Thakar S, Dadlani R, Tawari M, Hegde AS: Atypical cerebellar slump syndrome and external hydrocephalus following craniocervical decompression for Chiari I malformation: case report. Neurol Med Chir (Tokyo) 54:567-571, 2014

29. Tubbs RS, Beckman J, Naftel RP, Chern JJ, Wellons JC III, Rozzelle CJ, et al: Institutional experience with 500 cases of surgically treated pediatric Chiari malformation Type I. J Neurosurg Pediatr 7:248-256, 2011

30. Tubbs RS, Lyerly MJ, Loukas M, Shoja MM, Oakes WJ: The pediatric Chiari I malformation: a review. Childs Nerv Syst 23:1239-1250, 2007

31. Zakaria R, Kandasamy J, Khan Y, Jenkinson MD, Hall SR, Brodbelt A, et al: Raised intracranial pressure and hydrocephalus following hindbrain decompression for Chiari I malformation: a case series and review of the literature. $\mathbf{B r} \mathbf{J}$ Neurosurg 26:476-481, 2012

\section{Disclosures}

The authors report no conflict of interest concerning the materials or methods used in this study or the findings specified in this paper.

\section{Author Contributions}

Conception and design: Carey, Vivas, Shimony, Jallo. Acquisition of data: Vivas, Shimony, Jackson, Xu. Analysis and interpretation of data: Vivas, Shimony. Drafting the article: Carey, Vivas, Shimony, Xu, Jallo. Critically revising the article: Carey, Vivas, Shimony, Jackson, Jallo. Reviewed submitted version of manuscript: Vivas, Shimony, Jackson, Jallo, Rodriguez, Tuite. Approved the final version of the manuscript on behalf of all authors: Carey. Administrative/technical/material support: Vivas, Shimony. Study supervision: Carey, Vivas, Shimony, Jallo.

\section{Correspondence}

Carolyn M. Carey: Johns Hopkins All Children's Institute for Brain Protection Sciences, St. Petersburg, FL. ccarey12@jhmi. edu. 